\title{
Effects of a hops (Humulus lupulus L.) dry extract supplement on self-reported depression, anxiety and stress levels in apparently healthy young adults: a randomized, placebo-controlled, double-blind, crossover pilot study
}

\author{
Ioannis Kyrou, ${ }^{1,2,3,4}$ Aimilia Christou, ${ }^{1}$ Demosthenes Panagiotakos, ${ }^{1}$ \\ Charikleia Stefanaki, ${ }^{5}$ Katerina Skenderi, ${ }^{1}$ Konstantina Katsana, ${ }^{1}$ Constantine Tsigos $^{1}$
}

\begin{abstract}
${ }^{1}$ Department of Nutrition and Dietetics, School of Health Science and Education, Harokopio University, Athens, Greece, ${ }^{2}$ Aston Medical Research Institute, Aston Medical School, Aston University, B4 7ET, Birmingham, UK, ${ }^{3}$ Translational \& Experimental Medicine, Division of Biomedical Sciences, Warwick Medical School, University of Warwick, CV4 7AL, Coventry, UK, ${ }^{4}$ WISDEM, University Hospitals Coventry and Warwickshire NHS Trust, CV2 2DX, Coventry, UK, ${ }^{5}$ Choremeion Research Laboratory, $1^{\text {st }}$ Department of Pediatrics, Athens University Medical School, National and Kapodistrian University of Athens, Athens, Greece
\end{abstract}

\begin{abstract}
OBJECTIVE: The Humulus lupulus L. plant (hops) is used as a herbal medicinal product for anxiety/mood disorders. Our aim was to study the effects of a hops dry extract on self-reported depression, anxiety and stress levels in young adults. DESIGN: Apparently healthy young adults from our university completed the Depression Anxiety Stress Scale-21 (DASS-21) and those reporting at least mild depression, anxiety and stress were invited to complete the study intervention. This followed a randomized (1:1), placebo-controlled, double-blind, crossover design with two 4-week intervention periods (Melcalin hops or placebo; two 0.2 gr capsules once daily) separated by a 2-week wash-out. Anthropometric measurements, DASS-21 assessments and measurements of morning cortisol plasma levels were performed at the beginning and the end of the 4-week treatment periods. RESULTS: 36 participants (Females/Males: 31/5; age: 24.7 \pm 0.5 years) completed the study intervention (attrition: 6/42). No significant changes in body weight and composition or morning circulating cortisol were noted with the hops or placebo. Significantly decreased DASS-21 anxiety, depression and stress scores were documented with hops $(9.2 \pm 7.3$ vs. 5.1 $\pm 5.9,11.9 \pm 7.9$ vs. 9.2 \pm 7.4 , and 19.1 \pm 8.1 vs. 11.6 \pm 8.1 ; all $p$ values $<0.05)$, which were significantly greater compared to those caused by the placebo (all $p$ values $<0.05$ ). CONCLUSION: In otherwise healthy young adults reporting at least mild depression, anxiety
\end{abstract}


and stress symptoms, daily supplementation with a hops dry extract can significantly improve all these symptoms over a 4-week period. These beneficial effects agree with the indication of hops for anxiety/mood disorders and restlessness, as approved by the German Commission $\mathbf{E}$.

Key words: Anxiety, Depression, Humulus lupulus L, Melcalin hops supplement, Stress

\section{INTRODUCTION}

Chronic stress is frequently manifested with anxiety and/or depressive symptomatology, which progressively may lead to a spectrum of behavioral and/or somatic disorders. ${ }^{1-4}$ Currently, anxiety and mood disorders constitute the most prevalent mental health problems in the general population, which often coexist particularly in primary care settings and the community. ${ }^{5-7}$ Indeed, the lifetime prevalence of any mood disorder is estimated to average $12 \%$, whilst large population-based studies show that up to $33.7 \%$ of individuals in the general population are affected by an anxiety disorder in their lifetime. ${ }^{5,6}$ Moreover, as these stress-related disorders frequently affect young individuals during their most active years, there is also a significant impact on their quality of life and productivity, with the World Health Organization (WHO) recognizing depression as the leading cause of disability worldwide. ${ }^{8}$

In addition, anxiety disorders and depression have been traditionally amongst the most prominent reasons for using complementary therapies. ${ }^{9-11}$ Of note, such therapies are more common in patients reporting mental health-related problems than in the rest of the population, and they are also used more frequently compared to conventional therapies by individuals with self-defined anxiety disorders and severe depression. ${ }^{9}$ Thus, it is not surprising that most of the patients visiting mental health care providers for anxiety and/or mood disorders report the use of complementary therapies, with herbal medicinal products being used more frequently. ${ }^{10-12}$ Interestingly, the use of these therapies is increasing, not only because of dissatisfaction with conventional medicine, but also due to a holistic orientation to health, particularly regarding options to reduce stress/anxiety, and to a broader attitude towards seeking non-prescription and non-pharmacological treatments with fewer adverse effects. ${ }^{11-13}$
Amongst the plants used for medicinal purposes, the plant named Humulus lupulus L (Family: Cannabaceae) has a long-standing tradition of medicinal use in Europe. ${ }^{14}$ Hops is the common name of the strobiles (cones) of the female inflorescences of the Humulus lupulus L. plant and is widely used as a bittering agent in the beer brewing industry, as well as a herbal medicinal product mostly for sleep disturbances. ${ }^{15}$ Thus, hops in the form of herbal tea, infusions, liquid/alcoholic extracts, tinctures and powdered herbal substances have traditionally been used for the relief of insomnia, excitability and restlessness associated with tension headaches and nervous tension. ${ }^{14,15}$ Indeed, the German Commission E has approved the use of hops for sleep disturbances and mood disorders, such as anxiety and restlessness. ${ }^{16}$ Data from in vivo studies in rats have shown that a hops extract and its fraction containing alpha-bitter acids (humulones) exert significant sedative and antidepressant effects, whilst hops beta-acids (lupulones) appear to also exhibit antidepressant activity with fewer sedative effects, probably by affecting gamma-aminobutyric acid (GABA) neurotransmission activity. ${ }^{17,18}$ Moreover, in vitro binding experiments on selected central nervous system (CNS) receptors using a fixed combination valerian-hops extract and its individual components have shown that hops interact with certain serotonin $\left(5-\mathrm{HT}_{6}\right)$ and melatonin $\left(\mathrm{ML}_{1}\right)$ receptor subtypes, which are involved in various CNS functions related to stress activity, relaxation, circadian rhythms and sleep. ${ }^{19}$

Despite the popularity of hops, particularly in central Europe, as a sedative and mood-enhancing herbal medicinal product, there is a marked paucity of high-quality, placebo-controlled clinical studies on the efficacy of hops in reducing stress-related symptomatology. Therefore, the aim of the present study was to explore the effects of a commercially available hops dry extract on self-reported depression, anxiety and stress levels in apparently healthy young adults. 


\section{MATERIAL AND METHODS}

\section{Study protocol - Design}

The study was approved by the Institutional Review Board of Harokopio University, Athens, Greece, and was conducted in accordance with the principles and recommendations of the Declaration of Helsinki. ${ }^{20}$ The study protocol had a randomized, placebo-controlled, double-blind, crossover design with two 4-week intervention periods separated by a 2 -week wash-out period. Study participants were allocated to start the intervention with either placebo or hops dry extract capsules (two $0.2 \mathrm{~g}$ capsules once daily in the evening, which is the recommended daily dose for this hops supplement) according to a pre-established computer generated randomization schedule (randomization ratio: 1:1). Placebo and hops (0.2 g hops extract; Melcalin ${ }^{\circledR}$ HOPs, Biotekna Srl., Venice, Italy) capsules for the study treatment were kindly provided by Biotekna Srl. (Biotekna Srl., Venice, Italy). In the context of this study, placebo and hops capsules were provided in sealed, identical containers/bottles, each covering a 4-week treatment period. Each of these containers was coded as either "I" or "II" in order to blind both study investigators and participants to the randomization/ treatment until the study was completed. Each study participant was provided with one capsule container at the beginning of each intervention period, whilst all capsule containers were collected at the end of each 4-week period in order to count any unused capsules as a measure of compliance with the study treatment. For a 2-week period prior to the study initiation and throughout the total duration of the study, all participants were asked to abstain from hops-containing products (e.g. beer) and other supplements/vitamins (e.g. valerian or St. John's wort). In addition, all study subjects were asked to refrain from significant changes to their dietary habits and maintain their usual activity levels throughout the study duration. Moreover, all participants were also instructed to keep a record of any symptoms/signs of adverse events and/or illnesses, as well as any use of medications for the duration of the study, and these records were checked by the study investigators at the end of both experimental periods.

\section{Study participants}

The cohort of this study was recruited from young
Caucasian adults (age $>18$ years) attending Harokopio University, Athens, Greece at an under- or postgraduate level. The study exclusion criteria included any systemic disease (e.g. neurological or psychiatric disorders, including clinically diagnosed anxiety disorders and depression) or treatment (e.g. sedative or antidepressant medications and relevant supplements/preparations) which could interfere with the study objectives so that only apparently healthy adults were recruited into the study. Furthermore, individuals with recent (within the past two months) significant changes in body weight ( $>3 \%$ of total body weight) and/or physical activity levels as well as subjects with known drug and/or alcohol use disorders were excluded from recruitment. All study participants completed a psychological profile assessment via the validated, self-reported study questionnaire (i.e., the Depression Anxiety Stress Scale-21, as detailed in the following section) and volunteers with scores indicating at least mild depression, anxiety and stress levels were further invited to participate in the intervention part of the study. All study procedures were completed during an exam-free period to avoid bias relating to exam-related stress. Each participant was fully informed about the study aims, design and experimental procedures and provided written informed consent before participating in the study.

\section{Study protocol assessments}

\section{Anthropometry}

Anthropometric measurements were performed for all study subjects at the beginning and the end of the two 4-week treatment periods. Body weight and height were measured at our university research unit in participants without shoes or heavy clothing. Body weight (BW) was measured to the nearest 0.5 $\mathrm{kg}$ and height to the nearest $1 \mathrm{~cm}$. Body mass index (BMI) was calculated as body weight in kilograms divided by the square of the height in meters. Body composition analyses, including body fat mass (FM: $\% \mathrm{BW}$ ), total body water (TBW: \%BW) and extracellular water (ECW: \%BW), were also performed at the same time-points using a calibrated, standardized, dual frequency bioelectrical impedance analysis (BIA) instrument (BIA-ACC device, Biotekna Srl., Venice, Italy), as previously described. ${ }^{21}$ 
Assessments of self-reported depression, anxiety and stress symptomatology

Depression, anxiety and stress symptomatology was assessed in all study participants using the validated Greek translation of the Depression Anxiety Stress Scale-21 (DASS-21), which has been shown to be reliable in the Greek adult general population with psychometric properties similar to those reported in the international literature. ${ }^{22}$ The DASS- 21 is a wellestablished, validated, self-rating scale which measures the magnitude of three negative emotional states, i.e. depression, anxiety and stress. This instrument is considered both sensitive and accurate and thus is widely applied in research. Briefly, the DASS-21 consists of 21 self-reporting items with seven items in each of the three subscales (depression, anxiety, and stress, respectively), documenting the relevant symptomatology over the past week. ${ }^{23}$ Each item comprises a statement and four ordinal responses, which are rated as: (i) "Did not apply to me at all - NEVER"; (ii) "Applied to me to some degree, or some of the time - SOMETIMES"; (iii) "Applied to me to a considerable degree, or a good part of the time - OFTEN"; and (iv) "Applied to me very much, or most of the time - ALMOST ALWAYS", and are assigned a score of 0, 1, 2 and 3, respectively. Scores from the seven items in each subscale are summed up to yield a single subscale score. Each of these subscale scores is further multiplied by two (subscale score range: 0-42) in order to compare it with the normative data of the long form of the DASS instrument which has 42 items (DASS-42). Notably, the DASS-21 has the same factor structure and yields similar results to the DASS-42, while it requires half the time to be administered (5-10 mins). Subscale scores equal or higher than 10, 8 and 15 indicate the presence of at least mild depression, anxiety and stress symptomatology, respectively. Higher scores in each subscale indicate even greater severity of the corresponding depression, anxiety and stress symptoms. ${ }^{23}$

\section{Cortisol plasma levels measurements}

Blood sampling for measurements of morning cortisol plasma levels was performed in all study participants at the beginning and the end of the two 4-week intervention periods, as per study protocol. All venous blood samples were collected between
0800 and 0900 hours, after overnight fasting (10 hours of overnight fasting without food or drink intake, except for water). All plasma cortisol measurements were carried out on an AIA-600II analyzer (TOSOH Bioscience, Inc., South San Francisco, CA, USA) using a competitive fluorescence enzyme immunoassay based on individual test cups, according to the manufacturer's instructions (ST AIA-PACK CORT, TOSOH Bioscience, Inc., South San Francisco, CA, USA; assay range: $0.2-60 \mu \mathrm{g} / \mathrm{dL}$; intra- and inter-assay coefficient of variation $<5.0 \%$ ).

\section{Statistical analysis}

Normally distributed variables are presented as means \pm standard error (SEM), unless otherwise stated. Categorical variables are presented as frequencies. Differences between and within groups were evaluated using two-way (group X time) analysis of variance (ANOVA) and Repeated-Measures ANOVA, as appropriate. The Shapiro-Wilk test was used to evaluate the normality of the distribution of quantitative variables. Correlations between continuous variables with skewed distributions were tested by the Spearman's rank correlation coefficient. Associations between continuous and categorical variables were evaluated using the Pearson chi-square test and the Mann-Whitney test, where appropriate. Statistical significance was considered with a two-sided $p$ value of $<0.05$. The Statistical Package for the Social Sciences, SPSS, version 18.0 (SPSS Inc., Chicago, IL, USA) was used to analyze data.

\section{RESULTS}

Of the 126 adults who completed the DASS-2 1 questionnaire, 42 individuals (Females/Males: 38/4; age: $25 \pm 3.9$ years) were eligible based on the study inclusion/exclusion criteria and consented to participate in the intervention part of the study. Figure 1 presents the CONSORT flow diagram of all participants throughout the study stages. Six of the study subjects who entered the intervention part of the study failed to complete the first intervention arm as per protocol and were excluded from further analyses (Figure 1; study attrition rate: $14.2 \%$ ). None of the study participants experienced adverse/side effects from the study treatment (hops extract or placebo). 


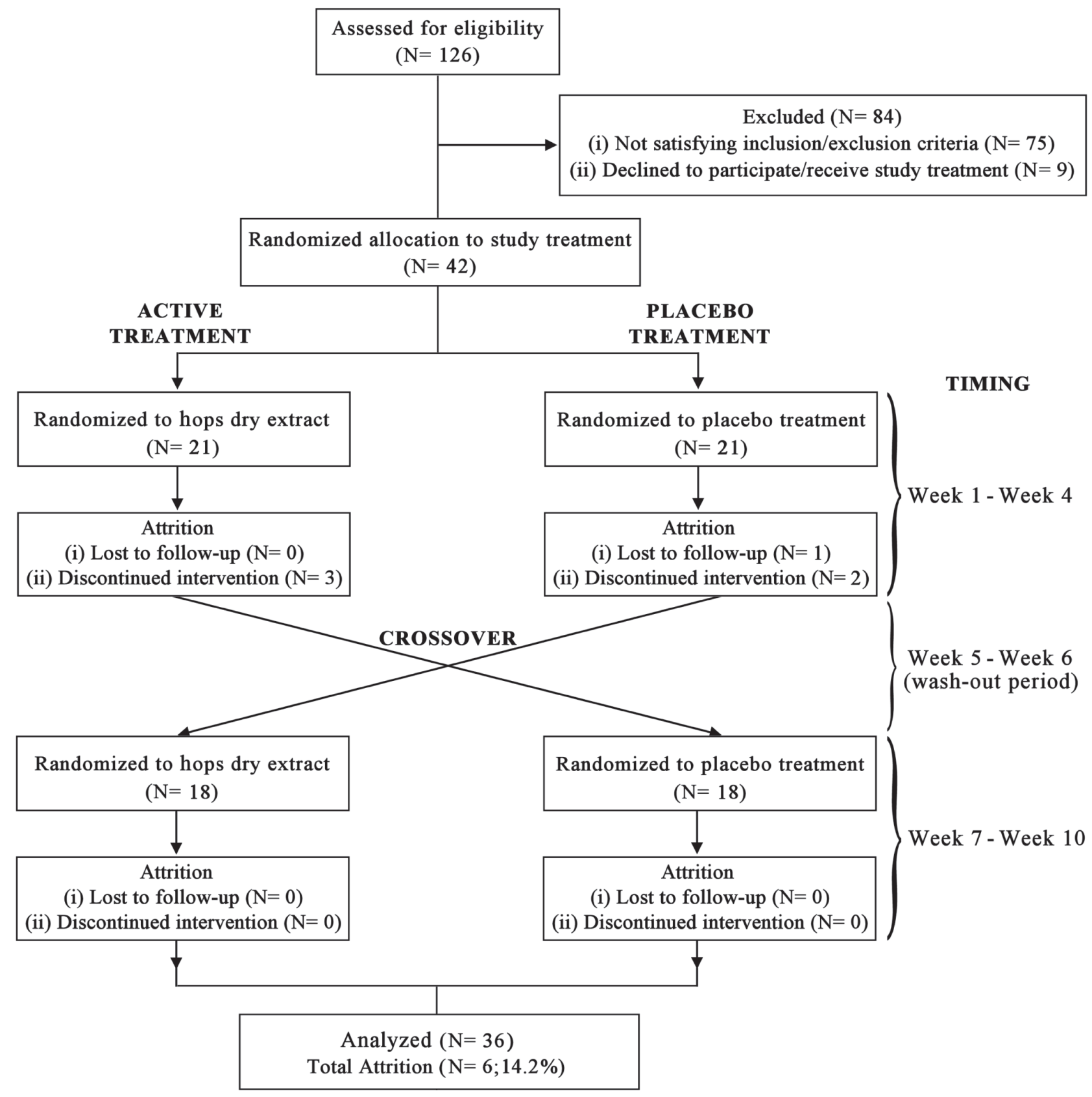

Figure 1. Flow diagram of all participants throughout the study stages.

The key baseline characteristics of the study participants $(N=36)$ who completed the two 4-week intervention periods (placebo and hops) of this doubleblind, randomized, crossover study are presented in Table 1 . The study participants exhibited no significant changes in body weight, BMI and body composition parameters (FM; TBW; ECW) with the two study treatments (data not shown; all corresponding $p$ values
$>0.05$ ). Similarly, neither the hops nor the placebo induced significant changes in the morning cortisol plasma levels in the study cohort $(17.7 \pm 5.8 \mu \mathrm{g} / \mathrm{dL} v s$. $17.6 \pm 6.3 \mu \mathrm{g} / \mathrm{dL}$ and $18.5 \pm 5.9 \mu \mathrm{g} / \mathrm{dL} v s .18 .9 \pm 4.8 \mu \mathrm{g} /$ $\mathrm{dL}$, respectively; both $p$ values $>0.05$ ).

A significant decrease in the DASS-21 anxiety score was noted with both the hops $(9.2 \pm 7.3$ vs. $5.1 \pm 5.9 ; p=0.003)$ and the placebo $(8.3 \pm 7.1 \mathrm{vs}$. 
Table 1. Selected baseline characteristics of the study participants $(N=36)$ who completed both the two 4-week intervention periods (placebo and hops) of this double-blind, randomized, crossover study

\begin{tabular}{lc}
\hline Baseline variable & Study cohort $(\mathbf{N = 3 6})$ \\
\hline Gender (Females/Males) & $31 / 5$ \\
Age (years) & $24.7 \pm 0.5$ \\
Body Weight $(\mathrm{kg})$ & $62.1 \pm 1.3$ \\
BMI $\left(\mathrm{kg} / \mathrm{m}^{2}\right)$ & $22.7 \pm 0.4$ \\
Fat Mass $(\% \mathrm{BW})$ & $23.8 \pm 0.8$ \\
Total Body Water $(\% \mathrm{BW})$ & $49.9 \pm 0.5$ \\
Extra Cellular Water $(\% \mathrm{BW})$ & $47.1 \pm 0.4$ \\
DASS-21 Depression Score & $11.9 \pm 1.0$ \\
DASS-21 Anxiety Score & $8.8 \pm 0.8$ \\
DASS-21 Stress Score & $18.4 \pm 1.1$ \\
Morning Plasma Cortisol $(\mu \mathrm{g} / \mathrm{dL})$ & $18.1 \pm 0.7$ \\
\hline
\end{tabular}

BMI: body mass index; BW: body weight; DASS-21: Depression anxiety stress scale-21; Data presented as means \pm standard error.

$6.2 \pm 5.6 ; p=0.041)$, which was significantly greater with the hops compared to the placebo $(p=0.009)$ (Figure 2). In addition, the DASS-21 depression score decreased significantly after the hops $(11.9 \pm 7.9$ vs. $9.2 \pm 7.4 ; p<0.001)$ and the placebo $(11.8 \pm 9.2 v s$. $10.7 \pm 8.6 ; p=0.005)$, with a significantly more potent effect following the hops treatment compared to placebo $(p=0.001)$ (Figure 3). Moreover, the DASS-21 stress scores were also significantly reduced with the hops and the placebo study treatments $(19.1 \pm 8.1$ vs. $11.6 \pm 8.1 ; p=0.003$, and $17.8 \pm 9.9$ vs. $15.1 \pm 8.8$; $p=0.022$, respectively), with hops again inducing a significantly greater effect than the placebo $(p=0.009)$ (Figure 4).

Finally, no correlations were identified between any of the three DASS-21 subscale scores and baseline or post-treatment study variables, including body weight, BMI, body composition parameters (FM; TBW; ECW) and morning cortisol plasma levels (data not shown).

\section{DISCUSSION}

The primary objective of this study was to explore potential effects of a commercially available hops dry extract on self-reported depression, anxiety and stress symptomatology in apparently healthy young adults.

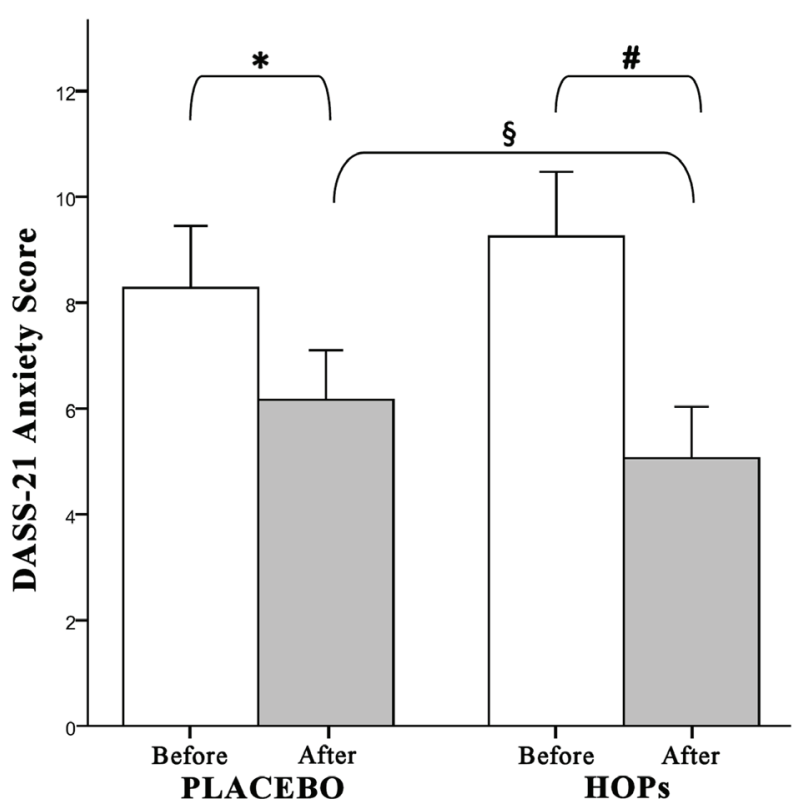

Figure 2. Depression anxiety stress scale-21 (DASS-21) anxiety scores at baseline and after hops and placebo 4-week treatment. Data are presented as means \pm standard error. *: $p=0.041$ before $v s$. after placebo treatment; $\#: p=0.003$ before $v s$. after hops treatment; $\S: p=0.009$ hops treatment $v s$. placebo treatment.

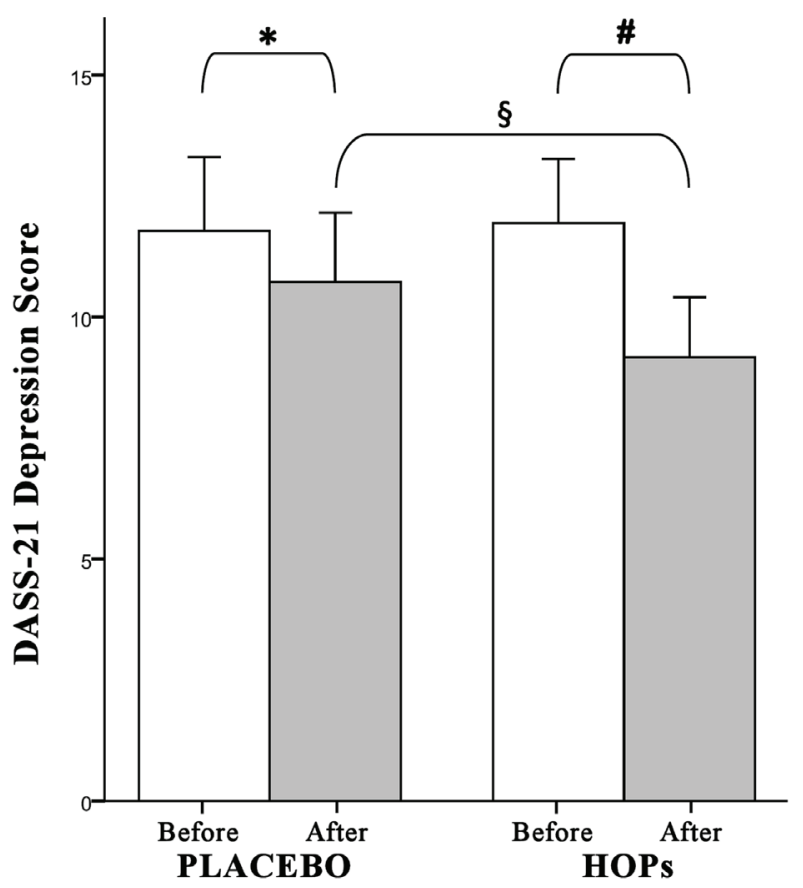

Figure 3. Depression anxiety stress scale-21 (DASS-21) depression scores at baseline and after hops and placebo 4-week treatment. Data are presented as means \pm standard error. $*: p=0.005$ before $v s$. after placebo treatment; \#: $p<0.001$ before vs. after hops treatment; $\S: p=0.001$ hops treatment $v s$. placebo treatment. 


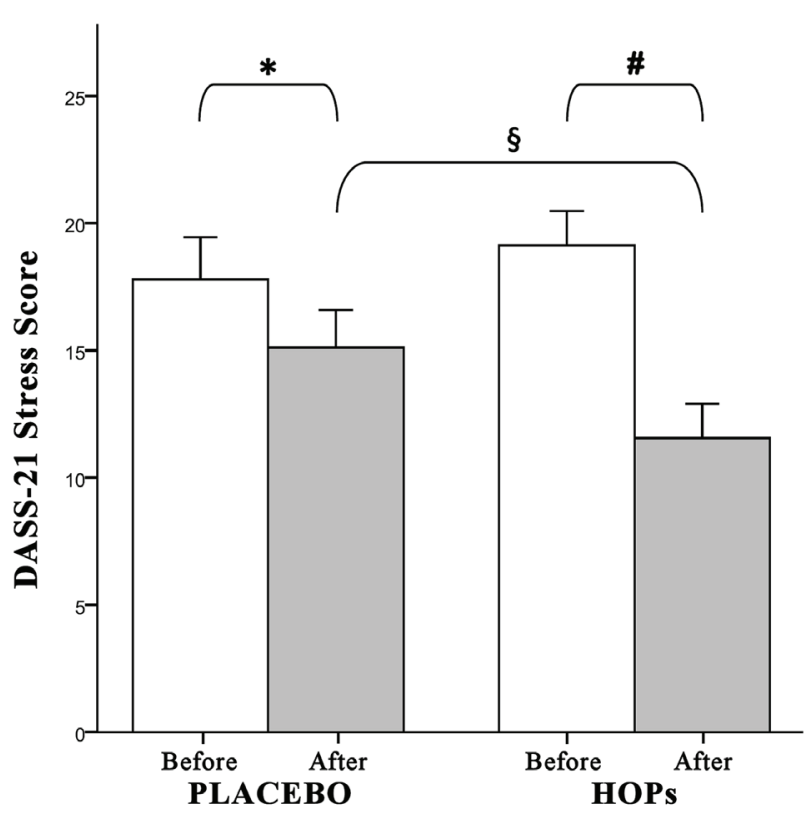

Figure 4. Depression anxiety stress scale-21 (DASS-21) stress scores at baseline and after hops and placebo 4-week treatment. Data are presented as means \pm standard error. *: $p=0.022$ before $v s$. after placebo treatment; $\#: p=0.003$ before $v s$. after hops treatment; $\S: p=0.009$ hops treatment $v s$. placebo treatment.

Applying a randomized, placebo-controlled, doubleblind, crossover study design, our findings in a cohort of otherwise healthy young adults with at least mild self-reported depression, anxiety and stress symptoms revealed a significant reduction in the levels of all these symptoms with hops which was significantly greater compared to placebo (Figures 2 to 4 ). These results are in accord with the traditional use and the indication of hops for mood disorders, anxiety and restlessness, as approved by the German Commission E. ${ }^{14,16}$ To date, most of the clinical data on the effects of hops in humans are from studies utilizing hops as part of a combination preparation, typically with valerian, ${ }^{14}$ and hence it is not feasible to draw safe conclusions regarding direct mood-enhancing effects of hops based on these studies. To the best of our knowledge, this is the first placebo-controlled clinical study showing that a hops extract alone can significantly improve symptoms relating to both depression and increased anxiety/stress, thus revealing these hops-specific beneficial effects.

Interestingly, it has been suggested in the literature that hops should be taken with caution by individu- als suffering from depression because of the known sedative effects of hops, which may accentuate depressive symptoms and potentiate the sedative effects of existing therapy. ${ }^{14,24}$ In regard to this point, our study findings suggest that hops may have an overall beneficial mood-enhancing effect without significant adverse/side effects in treatment-naïve individuals presenting with symptoms of both depression and anxiety/stress. Of note, this presence of depressive/ anxiety comorbidity is a frequent problem in everyday clinical practice, since anxiety and mood disorders often coexist, particularly in individuals in the general population. ${ }^{7}$ Additional clinical studies are required to further explore potential effects (beneficial and adverse/side effects) of hops in patients with depression not associated with increased anxiety/stress and in cases of anergic or atypical depression.

The pathophysiologic relationship between chronic stress and clinical manifestations of depression and/ or anxiety is complex, with melancholic depression representing a typical example of dysregulated stress response and hyperactivation of the stress system. ${ }^{1-4}$ Indeed, the stress system consists of a highly interconnected neuroendocrine infrastructure, which is located both in the CNS and the periphery. The central control stations of this system are positioned in the hypothalamus and the brain stem, including primarily the parvocellular corticotropin-releasing hormone (CRH) and arginine-vasopressin (AVP) neurons of the paraventricular hypothalamic nuclei, as well as the locus coeruleus/norepinephrine system. ${ }^{1}$ Furthermore, the hypothalamic-pituitary-adrenal (HPA) axis and the efferent sympathetic/adrenomedullary system constitute the main effector limbs via which the CNS regulates the adaptive stress responses. ${ }^{1}$ Based on the documented effects induced by the hops treatment in our study, it is plausible that hops may affect one or more of these stress system centers/components, thereby reducing the chronic stress-related over-activity and resulting in improvement of the manifested depression/anxiety symptoms. However, our study results showed no significant changes in the morning plasma levels of cortisol, which is the final hormonal effector of the HPA axis. ${ }^{1-4}$ This may suggest either that hops has no significant impact on basal circulating cortisol levels or that it may have more subtle effects on the circadian rhythm of the 
HPA axis and the diurnal variation of circulating cortisol. To identify such potential effects, further studies with a more complex study design would be required, utilizing multiple serial sampling of blood or saliva in order to explore hypothesized changes in the diurnal variation of cortisol levels.

Further underlying mechanisms may also be implicated in the effects of hops in the central stress system. Indeed, in vitro data indicate that hops may affect the GABA synthesis/metabolism and the GABAergic neurotransmission activity in the CNS..$^{15,18,25-28}$ Importantly, GABA is the key inhibitory neurotransmitter implicated in the regulation of neuronal excitability and central stress system activity, acting mostly as a brake on the stimulated neural circuitry during stress. ${ }^{1}$ Not surprisingly, low GABA levels in the CNS are associated with anxiety, restlessness and depression. ${ }^{29-32}$ Thus, hops could potentially exert anxiolytic and/or antidepressant effects by modulating the levels and/ or activity of GABA in the CNS. ${ }^{15,18,25-28}$ However, the evidence supporting this hypothesis is based on preliminary data from in vitro experimental studies, which need to be corroborated by in vivo and clinical data. Similarly, based on in vitro binding experiments hops appears to interact with both serotonin $\left(5-\mathrm{HT}_{6}\right)$ and melatonin $\left(\mathrm{ML}_{1}\right)$ receptor subtypes in the $\mathrm{CNS},{ }^{19}$ hence its anxiolytic and/or antidepressant effects may also involve the corresponding neural circuits, which have a well-established role in depression and the regulation of the central stress system and circadian rhythms. ${ }^{1,28}$

Finally, another mechanism that may contribute to the hops-induced effects noted in our study is improvement in the sleep pattern/quality due to the sedative activity of hops. ${ }^{14,15}$ This has also been suggested in two studies by Franco et al. which used non-alcoholic beer as a dietary source of hops over a 2-week period in 30 university students and 17 healthy female nurses, respectively. ${ }^{33,34}$ Both these studies reported improvement in night sleep quality (e.g. reduced sleep latency), with the latter study suggesting that the effects on sleep quality were also reflected in decreased self-reported anxiety levels, as measured by the State-Trait Anxiety Inventory. ${ }^{34}$ However, these two studies were not conducted in a blinded manner, lacked a placebo-controlled arm and had a non-standardized source of hops, thus limiting the interpretation and implications of the reported findings, particularly regarding the hops effects on anxiety levels through improved sleep quality. In order to explore this hypothesis, welldesigned, placebo-controlled studies with subjective and objective measures of sleep quality, quantity and architecture will be required.

\section{LIMITATIONS}

A limitation of this study is that the depression, anxiety and stress symptomatology of the participants was self-reported. However, the DASS-21 is a wellvalidated instrument, which is shown to accurately capture the underlying symptoms of these emotional states and is widely applied in this type of research. ${ }^{23}$ Furthermore, complimentary/herbal medicines are used more frequently by individuals with self-defined anxiety and mood disorders, ${ }^{9}$ thus our study cohort represents a highly relevant population of particular research interest appropriate for exploring the effects of a commercially available, non-prescription hops dry extract supplement on depression and anxiety/ stress symptoms.

In this study, a random sample from the under- and post-graduate students at our university was recruited. A recruitment/selection bias may thus have existed in the recruitment process of our study cohort, as the study was advertised only to our student body. Such limitations are relatively common in this type of research, yet it was also our intention to explore this specific intervention in young adults with undiagnosed depression and anxiety/stress symptomatology, as the consequences of untreated anxiety and mood disorders in this age group are profound on both quality of life and productivity. Of note, our study was conducted in an exam-free period of the academic year, so that our study cohort can be considered at least to an adequate degree representative of the Greek general population of otherwise healthy young adults.

It should also be noted that in our study we documented a significant effect of the placebo in decreasing the self-reported depression, anxiety and stress levels. These placebo effects may be documented in the context of such interventions to treat depression and/or anxiety/stress disorders, especially since anxiety reduction and release of 
endogenous opioids (e.g. dopamine and endorphins) are considered among the putative mechanisms mediating the placebo effect(s). ${ }^{35-38}$ However, despite the noted placebo effects, our study findings have allowed us to identify the significantly greater effects of the hops dry extract used as compared to placebo. This further highlights the importance of blinded, placebo-controlled study designs, particularly in studies focused on anxiety/depression and/ or complementary medicine. Notably, it has been suggested that a "balanced crossover design" may be applied in order to further address issues relating to interactions between drug-specific and unspecific effects; however, this design has practical disadvantages (i.e. it requires four study groups and thus an increased number of participants) and raises certain ethical issues (e.g. in this study design, in addition to the two typical crossover study groups, one study group receives the drug twice and another group receives the placebo twice instead of switching from the study drug to placebo or vice versa). ${ }^{39,40}$

Finally, our study assessments included profiling only of morning cortisol plasma levels which did not detect significant changes with the study intervention. In order to further explore potential effects of hops supplementation on the diurnal variation of stress system activity, additional studies would be required with serial sampling of blood and/or saliva to assess any changes in relevant biomarkers, such as salivary alpha-amylase (a non-invasive biomarker for the sympathetic nervous system activity). ${ }^{41}$

\section{CONCLUSION}

Our study presents novel data showing that daily supplementation with a commercially available hops dry extract for a 4-week period in a cohort of otherwise healthy young adults with at least mild self-reported depression, anxiety and stress symptoms can significantly reduce the levels of all these symptoms. This finding adds to the existing literature on the hopsinduced effects, since most of the existing clinical studies have used combinations of hops with other sedative plant extracts. Longer studies are required to explore the long-term efficacy and safety of this intervention, which should be also studied in older patients with depression and/or anxiety/stress disorders.

\section{CONFLICT OF INTEREST}

None

\section{REFERENCES}

1. Tsigos C, Kyrou I, Kassi E, Chrousos GP, 2016 Stress, endocrine physiology and pathophysiology. In: De Groot LJ, Chrousos G, Dungan K, et al, (eds) Endotext [Internet]. South Dartmouth (MA): MDText.com, Inc.

2. Chrousos GP, 2009 Stress and disorders of the stress system. Nat Rev Endocrinol 5: 374-381.

3. Charmandari E, Tsigos C, Chrousos G, 2005 Endocrinology of the stress response. Annu Rev Physiol 67: 259-284.

4. Kyrou I, Tsigos C, 2007 Stress mechanisms and metabolic complications. Horm Metab Res 39: 430-438.

5. Kessler RC, Aguilar-Gaxiola S, Alonso J, et al, 2009 The global burden of mental disorders: an update from the WHO World Mental Health (WMH) surveys. Epidemiol Psichiatr Soc 18: 23-33.

6. Bandelow B, Michaelis S, 2015 Epidemiology of anxiety disorders in the 21st century. Dialogues Clin Neurosci 17: 327-335.

7. Hirschfeld RM, 2001 The comorbidity of major depression and anxiety disorders: recognition and management in primary care. Prim Care Companion J Clin Psychiatry 3: 244-254.

8. World Health Organization (WHO), 2016 Fact sheet on depression. Geneva, Online at: www.who.int/mediacentre/factsheets/fs369/en/ [Last accessed 27 May 2017].

9. Kessler RC, Soukup J, Davis RB, et al, 2001 The use of complementary and alternative therapies to treat anxiety and depression in the United States. Am J Psychiatry 158: 289-294.

10. van der Watt G, Laugharne J, Janca A, 2008 Complementary and alternative medicine in the treatment of anxiety and depression. Curr Opin Psychiatry 21:37-42.

11. Ernst E, Rand JI, Stevinson C, 1998 Complementary Therapies for depression an overview. Arch Gen Psychiatry 55: 1026-1032.

12. Astin JA, 1998 Why patients use alternative medicine: results of a national study. JAMA 279: 1548-1553.

13. Ernst E, Wiloughby M, Weihmayr T, 1995 Nine possible reasons for choosing complementary medicine. Perfusion 11: 356-359.

14. EMA, 2014 Assessment report on Humulus lupulus L., flos. EMA/HMPC/418902/2005. 2014. Online at: www.ema.europa.eu/docs/en_GB/document_library/Herbal___HMPC_assessment_report/2014/08/ WC500170935.pdf [Last accessed 27 May 2017].

15. Zanoli P, Zavatti M, 2008 Pharmacognostic and pharmacological profile of Humulus lupulus L. J Ethnopharmacol 116: 383-396.

16. Blumenthal M, Goldberg A, Brinckmann J, (eds) 2000 
Herbal medicine: Expanded commission E monographs, first ed. Integr Med Comm, Newton, 193-196.

17. Zanoli P, Rivasi M, Zavatti M, Brusiani F, Baraldi M, 2005 New insight in the neuropharmacological activity of Humulus lupulus L. J Ethnopharmacol 102: 102-106.

18. Zanoli P, Zavatti M, Rivasi M, et al, 2007 Evidence that the beta-acids fraction of hops reduces central GABAergic neurotransmission. J Ethnopharmacol 109: 87-92.

19. Abourashed EA, Koetter U, Brattström A, 2004 In vitro binding experiments with a Valerian, hops and their fixed combination extract (Ze91019) to selected central nervous system receptors. Phytomedicine 11: 633-638.

20. World Medical Association (WMA), 2013 Declaration of Helsinki. Amended by the 64th WMA General Assembly, Fortaleza, Brazil, WMA Archives, FerneyVoltaire, France. WMA Declaration of Helsinki - Ethical Principles for Medical Research Involving Human Subjects.

21. Tsigos C, Stefanaki C, Lambrou GI, Boschiero D, Chrousos GP, 2015 Stress and inflammatory biomarkers and symptoms are associated with bioimpedance measures. Eur J Clin Invest 45: 126-134.

22. Lyrakos GN, Arvaniti C, Smyrnioti M, Kostopanagiotou G, 2011 Translation and validation study of the depression anxiety stress scale in the Greek general population and in a psychiatric patient's sample. Eur Psychiatry 26: Suppl 1: 1731.

23. Lovibond SH, Lovibond PF, 1995 Manual for the depression anxiety stress scales (2nd ed.); Sydney: Psychology Foundation.

24. Ernst E, Pittler MH, Stevinson C, White A (eds), 2001 The desktop guide to complementary and alternative medicine: An Evidence-Based Approach. St Louis: Mosby.

25. Aoshima H, Takeda K, Okita Y, Hossain SJ, Koda H, Kiso Y, 2006 Effects of beer and hop on ionotropic gamma-aminobutyric acid receptors. J Agric Food Chem 54: 2514-2519.

26. Meissner O, Häberlein H, 2006 Influence of xanthohumol on the binding behavior of GABAA receptors and their lateral mobility at hippocampal neurons. Planta Med 72: 656-658.

27. Awad R, Levac D, Cybulska P, Merali Z, Trudeau VL, Arnason JT, 2007 Effects of traditionally used anxiolytic botanicals on enzymes of the gamma-aminobutyric acid (GABA) system. Can J Physiol Pharmacol 85: 933-942.

28. Weeks BS, 2009 Formulations of dietary supplements and herbal extracts for relaxation and anxiolytic action: Relarian. Med Sci Monit 15: RA256-262.

29. Nuss P, 2015 Anxiety disorders and GABA neurotransmission: a disturbance of modulation. Neuropsychiatr Dis Treat 11: 165-175.

30. Nemeroff CB, 2003 The role of GABA in the pathophysiology and treatment of anxiety disorders. Psychopharmacol Bull 37: 133-146.

31. Kendell SF, Krystal JH, Sanacora G, 2005 GABA and glutamate systems as therapeutic targets in depression and mood disorders. Expert Opin Ther Targets 9: 153168.

32. Möhler H, 2012 The GABA system in anxiety and depression and its therapeutic potential. Neuropharmacology 62: 42-53.

33. Franco L, Bravo R, Galán C, Rodríguez AB, Barriga C, Cubero J, 2014 Effect of non-alcoholic beer on Subjective Sleep Quality in a university stressed population. Acta Physiol Hung 101: 353-361.

34. Franco L, Sánchez C, Bravo R, et al, 2012 The sedative effect of non-alcoholic beer in healthy female nurses. PLoS One 7: e37290.

35. Haour F, 2005 Mechanisms of the placebo effect and of conditioning. Neuroimmunomodulation 12: 195-200.

36. Ernst E, 2001 Towards a scientific understanding of placebo effects. In Peters D (ed) Understanding the placebo effect in complementary medicine. London, UK: Churchill Livingstone; pp, 17-29.

37. Klosterhalfen S, Enck P, 2006 Psychobiology of the placebo response. Auton Neurosci 125: 94-99.

38. Ernst E. 2007 Placebo: new insights into an old enigma. Drug Discov Today 12: 413-418.

39. Enck P, Klosterhalfen S, Weimer K, Horing B, Zipfel S, 2011 The placebo response in clinical trials: more questions than answers. Philos Trans R Soc Lond B Biol Sci 366: 1889-1895.

40. Kube T, Rief W, 2017 Are placebo and drug-specific effects additive? Questioning basic assumptions of double-blinded randomized clinical trials and presenting novel study designs. Drug Discov Today 22: 729-735.

41. Nater UM, Rohleder N, 2009 Salivary alpha-amylase as a non-invasive biomarker for the sympathetic nervous system: current state of research. Psychoneuroendocrinology 34: 486-496. 